\title{
One-Pot Process of Naphthoquinones Synthesis from Hydroquinone in the Presence of Solutions of Mo-V-P Heteropolyacids as Bifunctional Catalysts
}

\author{
Leonid L. Gogin*, Elena G. Zhizhina, Zinaida P. Pai \\ Federal Research Center Boreskov Institute of Catalysis, Novosibirsk, Russia \\ Email: *gogin@catalysis.ru
}

How to cite this paper: Gogin, L.L., Zhizhina, E.G. and Pai, Z.P. (2019) One-Pot Process of Naphthoquinones Synthesis from Hydroquinone in the Presence of Solutions of Mo-V-P Heteropolyacids as Bifunctional Catalysts. Modern Research in Catalysis, $\mathbf{8}$, 1-9.

https://doi.org/10.4236/mrc.2019.81001

Received: November 30, 2018

Accepted: January 28, 2019

Published: January 31, 2019

Copyright $\odot 2019$ by author(s) and Scientific Research Publishing Inc. This work is licensed under the Creative Commons Attribution International License (CC BY 4.0).

http://creativecommons.org/licenses/by/4.0/

\begin{abstract}
1,4-Naphthoquinones (NQ) is a class of organic compounds with a number of important properties. However, the existing methods of their synthesis either give a low NQ yield or multistage and require a lot of time. In the present work new one-pot process of NQ synthesis from hydroquinone (HQ) and substituted 1,3-dienes at room temperature is proposed. The solution of heteropolyacid $\mathrm{H}_{7} \mathrm{PMo}_{8} \mathrm{~V}_{4} \mathrm{O}_{40}$ (HPA-4) is used as a bifunctional (acid and redox) catalyst for this process. The influence of a number of factors on key process parameters has been studied. It is demonstrated that unsubstituted and a number of alkyl substituted NQ with the yields of 50\%-80\% and purity of $92 \%-99 \%$ can be obtained by this method. The results obtained allow to significantly simplify the synthesis of NQ directly from HQ in comparison with the described similar methods.
\end{abstract}

\section{Keywords}

Hydroquinone, Naphthoquinones, Heteropolyacid, Diene Synthesis

\section{Introduction}

1,4-Naphthoquinones (henceforth NQ) is a class of organic compounds with a number of important properties. The unsubstituted NQ is used for the synthesis of 9,10-anthraquinone (in particular, Kawasaki, Japan, manufactures up to 3000 tons of anthraquinone from 1.4-NQ per year), a number of NQ are the regulators of polymerization, inhibitors of corrosion, and stabilizers of transformer oils [1]. The chlorinated NQ are used in the manufacturing of dyes [1]. Many NQ 
are found in nature and show various biological activities, for instance, antiviral, antifungal, vitamin, antimicrobial and antiparasitic, antitumor, immunomodulating, and hemostatic [2] [3].

In industry the elementary NQ are synthesized by oxidizing the corresponding naphthalenes [1]. For instance, oxidation of naphthalene by air oxygen on the vanadium catalyst is used to synthesize the unsubstituted NQ. The yield of NQ is only 36\%, and the main side product is phthalic anhydride. 2-Methyl-1,4-naphthoquinone (vitamin $\mathrm{K}_{3}$ ) is synthesized by oxidizing 2-methylnaphthalene by chromic mixture [1]. In addition to the mentioned oxidizers, the following substances can be used as such: chromic acid, lead dioxide, ammonium persulphate, ions of cerium (IV) [1]. However, in the first case, the yield of NQ is low and a significant amount of phthalic anhydride forms, and other oxidizers are either toxic, or produce huge wastes.

Still another way of NQ production is the diene synthesis between p-benzoquinone (BQ) and 1,3-dienes. The obtained adducts can be further oxidized into NQ by various oxidizers in an acid medium $\left(\mathrm{CuCl}_{2}, \mathrm{H}_{2} \mathrm{O}_{2}, \mathrm{NaClO}_{3}\right.$, $\mathrm{K}_{2} \mathrm{Cr}_{2} \mathrm{O}_{7}$ [4]) or by air oxygen in alkaline medium [5]. However, this method requires a rather long time to be completed. The works [3] [6] describe the synthesis of 6-methyl-NQ from BQ and isoprene with the following oxidation of adduct of diene synthesis by potassium or sodium bichromate in acid medium. The process takes more than 72 hours (yield of 6-methyl-NQ from BQ was $71 \%)$. In the case of unsubstituted NQ only synthesis of Diels-Alder adduct requires more than $48 \mathrm{~h}$. Oxidation of the adduct by $\mathrm{CrO}_{3}$ gives NQ with total yield of $66 \%$ [7].

We have studied the one-pot synthesis of 9,10-anthraquinone (AQ) and its derivatives from unsubstituted NQ and 1,3-dienes in the presence of high-vanadium solutions of heteropolyacids with empirical formulas $\mathrm{H}_{15} \mathrm{P}_{4} \mathrm{Mo}_{18} \mathrm{~V}_{7} \mathrm{O}_{89}$ (HPA-7) and $\mathrm{H}_{17} \mathrm{P}_{3} \mathrm{Mo}_{16} \mathrm{~V}_{10} \mathrm{O}_{89}$ (HPA-10) and hydrophilic organic solvent (1,4-dioxane) [8]. In this process the solutions of HPA-x that are both strong Brønsted acids and quite strong oxidizers due to the presence of oxocations $\mathrm{V}(\mathrm{V})$ play the role of bifunctional catalysts: the acid catalysts of diene synthesis and catalysts of oxidation. This allows a combination of both successive reactions in one technological stage without isolation of adducts of diene synthesis. Thus, in the complete conversion of NQ, the yield of AQ at $80^{\circ} \mathrm{C}$ reached $70 \%$. The slightlysoluble product of the reaction precipitated. It is important to note that the content of the main substance (AQ) in the precipitate was about $97 \%$.

It is known that for the diene synthesis of AQ not only NQ can be used as the initial substrate but also BQ [9]. According to our data, BQ can be quantitatively synthesized by oxidating hydroquinone (HQ) in the solutions of HPA-x in mild conditions (for comparison, oxidation of HQ by potassium bichromate gives BQ with a yield of $80 \%$ [10]). In [11] it was shown that in the presence of solutions of HPA-x (where $\mathrm{x}$ is the number of atoms of $\mathrm{V}$ within the HPA composition) the reaction between HQ and excessive butadiene in the absence of organic solution depending on temperature leads to the mixtures containing NQ, AQ, as well 
as hydrated derivatives of $\mathrm{AQ}$ in various ratios. At room temperature, NQ prevails in the mixture (up to $70 \%$ ), whereas at the elevated temperatures it is AQ and its hydrated derivatives. Therefore it is logical to expect the formation of NQ at interaction of HQ and 1,3-dienes in the molar ratio close to 1:1 in the presence of solutions of HPA-x at room temperature.

This work is dedicated to the study of the possible realization of one-pot synthesis of a number of NQ from HQ and 1,3-dienes in the presence of solutions Mo-V-P HPA-x as bifunctional catalysts. Note that before our works the methods of one-pot synthesis of NQ from HQ have not been described.

\section{Experimental}

\subsection{Reagents}

For naphthoquinones synthesis we used high purity hydroquinone (no less than 99\% main substance content, GOST 19627-74, Russia), 1,3-butadiene (99\% main substance content, Omsk Oil Refinery Plant, Russia), isoprene (99\%, Sigma Aldrich), 2,3-dimethyl butadiene (98\%, Alfa Aesar), trans-piperylene (90\%, Sigma Aldrich), 1,3-cyclohexadiene (96\%, Acros Organics), 1,4-dioxane (99\%, Fluka).

\subsection{Synthesis of HPA-x Solutions}

In experiments we used $0.25 \mathrm{~mol} \cdot \mathrm{L}^{-1}$ solutions of HPA-4 $\left(\mathrm{H}_{7} \mathrm{PMo}_{8} \mathrm{~V}_{4} \mathrm{O}_{40}, \mathrm{M}=\right.$ 1650), HPA-7 $\left(\mathrm{H}_{15} \mathrm{P}_{4} \mathrm{Mo}_{18} \mathrm{~V}_{7} \mathrm{O}_{89}, \mathrm{M}=3648\right)$ and HPA-10 $\left(\mathrm{H}_{17} \mathrm{P}_{3} \mathrm{Mo}_{16} \mathrm{~V}_{10} \mathrm{O}_{89}, \mathrm{M}=\right.$ 3580), synthesized as described elsewhere [12]. HPA-10 is the strongest oxidant among Mo-V-P heteropolyacids. For $\mathrm{x}<4$ there is mainly a reaction of diene synthesis.

\subsection{Analysis of Reaction Products}

Reaction products were analyzed with HPLC. For the purpose, we used liquid chromatograph Pro Star, equipped with Pro Star 410 Auto-Sampler, Pro Star 210, Pro Star 218 Solvent Delivery Module, Varian 500-LC Column Valve Module and UV-detector Photodiode Pro Star 335 (wavelength $247 \mathrm{~nm}$ ). Products were separated using Pursuit $3 \mathrm{C} 18$ column $(247 \times 4.6 \mathrm{~mm})$, the eluent rate was 1 $\mathrm{mL} / \mathrm{min}$. For chromatography, we used the following solvents: methanol (J. T. Baker, 99\%, UV-IR-HPLC); trifluoroacetic acid (Acros Organics, 99\%, chemical purity grade) and deionized water. Eluent composition was $70 \% \mathrm{CH}_{3} \mathrm{OH}+30 \%$ $\mathrm{CF}_{3} \mathrm{COOH}$ (samples solvent-chloroform).

The end of 6-methyl-NQsynthesis was controlled also by TLC (Sorbfil plates, eluent $\mathrm{CHCl}_{3}$ : benzene 1:1, absence of $\mathrm{HQ}$ and $\mathrm{BQ}$, comparison with known samples of NQ). Individual substituted NQ were identified by their melting points [6] [13], analysis of its IR spectra [6] and gas chromatography-mass spectrometry data (GCMS). IR spectra were recorded using spectrometer Shimadzu IR-Affinity-1, GC-MS ones were recorded using spectrometer Shimadzu GCMS-QP2010 Ultra.

Below are the physical data of prepared NQ and product of reaction between HQ and cyclohexadiene: 
1,4-Naphthoquinone. Yellow-brown solid, m.p. $122^{\circ} \mathrm{C}-123^{\circ} \mathrm{C}$, lit. [13] m.p. $125^{\circ} \mathrm{C}$. IR(KBr): $1661 \mathrm{~cm}^{-1}(\mathrm{C}=\mathrm{O})$; MS: $m / z 158$.

5-Methyl-1,4-naphthoquinone. Yellow solid, m.p. $119^{\circ} \mathrm{C}-121^{\circ} \mathrm{C}$, lit. [13] m.p. $121^{\circ} \mathrm{C}-122^{\circ} \mathrm{C}$. IR(KBr): $1659 \mathrm{~cm}^{-1}(\mathrm{C}=\mathrm{O})$; MS: $\mathrm{m} / z 172$.

6-Methyl-1,4-naphthoquinone. Yellow solid, m.p. $89^{\circ} \mathrm{C}-91^{\circ} \mathrm{C}$, lit. [6] m.p. $90^{\circ} \mathrm{C}-91^{\circ} \mathrm{C}$. IR(KBr): $1664 \mathrm{~cm}^{-1}(\mathrm{C}=\mathrm{O})$; MS: $\mathrm{m} / z 172$.

6,7-Dimethyl-1,4-naphthoquinone. Yellow solid, m.p. $116^{\circ} \mathrm{C}-119^{\circ} \mathrm{C}$, lit. [13] m.p. $118^{\circ} \mathrm{C}-119^{\circ} \mathrm{C}$. IR(KBr): $1667 \mathrm{~cm}^{-1}(\mathrm{C}=\mathrm{O})$; MS: $\mathrm{m} / z 186$.

Product of the reaction between $H Q$ and cyclohexadiene. Yellow solid. IR(KBr): $1664 \mathrm{~cm}^{-1}(\mathrm{C}=\mathrm{O})$; MS: $\mathrm{m} / z 188$ (main) and 238.

\subsection{Procedure of NQ Syntheses}

NQ syntheses were performed under atmospheric pressure and room temperature in a glass reactor (a volume of $100 \mathrm{~mL}) .0 .22 \mathrm{~g}(0.002 \mathrm{~mol})$ of HQ were put in a reactor, then mixed with organic solvent (1,4-dioxane) on stirring. After HQ dissolving the required amounts of $0.25 \mathrm{~mol} \cdot \mathrm{L}^{-1} \mathrm{HPA}-\mathrm{x}$ solution and diene were introduced (for 1,3-butadiene the reaction was carried out in its atmosphere and in a reactor of $50 \mathrm{~mL}$ too; see the Tables). All synthesis procedures were performed under intensive agitation with a magnetic stirrer $\left(650 \mathrm{~min}^{-1}\right)$ for 6 - 30 hours. In the synthesis course, HPA-x color changed from dark red to green one, and poorly soluble sediment of reaction products precipitated from the solution.

After process completion, solid precipitate was then filtered away, washed with water to neutral reaction and dried in vacuum over $\mathrm{P}_{2} \mathrm{O}_{5}$. The filtrate contained some quantity of reaction product was extracted with $3 \times 30 \mathrm{~mL}$ ether; the extract was dried over $\mathrm{CaCl}_{2}$. The solvent was distilled off on a rotary evaporator.

The combined dried precipitate was weighted and analyzed with HPLC.

NQ yield (in \% of the theoretically possible) was calculated via the formula:

$$
\mathrm{Y}_{\mathrm{NQ}}=\left(\mathrm{M} / \mathrm{Y}_{\mathrm{t}}\right) \times \mathrm{C}_{\mathrm{NQ}} \times 100 \text {, }
$$

where $\mathrm{M}$ was dry product mass, $\mathrm{g}$; $\mathrm{Y}_{\mathrm{t}}$ was theoretically possible yield of NQ at total HQ conversion, $\mathrm{g} ; \mathrm{C}_{\mathrm{NQ}}$ was NQ portion in solid product according to analysis results, \%.

After dioxane distillation, the boiling point of its azeotrope with water being $88^{\circ} \mathrm{C}$, and water excess evaporation reduced HPA-x solutions were regenerated by oxygen according to the method described in [8].

\section{Results and Discussion}

In this work we studied the reactions between HQ and various 1,3-dienes that proceed according to the following Scheme 1.

Here, HQ, hydroquinone; BQ, p-benzoquinone; THNQ, substituted tetrahydro-1,4-naphthoquinone; DHNQ, substituted dihydro-1,4-naphthoquinone; NQ, substituted 1,4-naphthoquinone. $\mathrm{R}_{1}=\mathrm{H}, \mathrm{CH}_{3} ; \mathrm{R}_{2}=\mathrm{H}, \mathrm{CH}_{3} ; \mathrm{R}_{3}=\mathrm{H}, \mathrm{CH}_{3}$. 


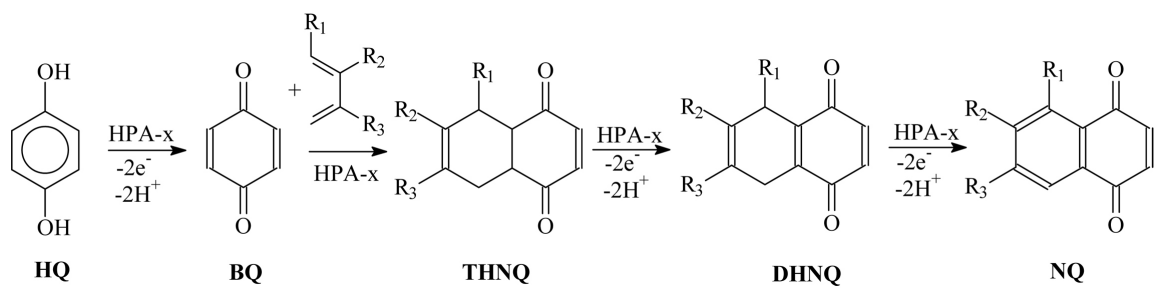

Scheme 1. NQ synthesis from HQ.

In addition, we studied the reaction of HQ with 1,3-cyclohexadiene proceeding along the supposed Scheme 2.

Here, I, 1a,4a-dihydro-5,8-endoethylene-1,4-naphthoquinone; II, 5,8-endoethylene-1,4-naphthoquinone.

For specification of the best conditions for the realization of the one-pot processes we chose the interaction of HQ with isoprene $\left(\mathrm{R}_{1}=\mathrm{CH}_{3} ; \mathrm{R}_{2}=\mathrm{H}\right)$, and not with the gaseous 1,3-butadiene considering the simpler control of reagents dosing.

In the first series of experiments we varied the number of vanadium atoms within HPA-x $(\mathrm{x}=4,7,10)$, i.e. changed the concentration of $\mathrm{VO}_{2}^{+}$, the main oxidizing particle in the solutions HPA-x. With that, the value of redox-potential of solution $E$ also changed. The obtained results are given in Table 1 .

The data in Table 1 show that the yield of the target 6-methyl-NQ is somewhat higher in the case of high-vanadium HPA-7 and HPA-10 than in the case of HPA-4, however, the purest product is obtained in the presence of HPA-4. According to the HPLC and GC-MS data, in the case of high-vanadium HPA-x, the products featured a noticeable admixture of the derivatives of anthraquinone. That means that in the presence of the solutions HPA-7 and HPA-10 with higher $E$ values, the one-pot process ran deeper, and it was the adducts of diene synthesis of NQ and isoprene that were subject to oxidation. Since for synthesis of substituted NQ HPA-4 was enough, the further studying of synthesis of 6-methyl-NQ was conducted with HPA-4 and not with the high-vanadium solutions.

We estimated the possibility to perform the reaction of HQ with isoprene in the absence of organic solvent. It was discovered that in this case the reaction product has a significant admixture (up to $20 \%$ ) of underoxidized (partially hydrated) derivatives of NQ as well as about $1 \%$ of unreacted BQ (Table 2). Obviously, the absence of organic solvent results in the problem of mixing the phases, and the reaction rate drops due to the diffusion limitations. Further studying of the processes was conducted in the presence of 1,4-dioxane as the hydrophilic organic solvent.

For choosing the best time of the one-pot process we studied the dependence of its parameters on time. It was found out that after $6 \mathrm{~h}$ of the reaction the main product of the interaction of HQ with isoprene is 6-methyl-DHNQ. After $24 \mathrm{~h}$ of the reaction the product still has a slight admixture of hydrogenated derivatives of NQ (Table 3), after $30 \mathrm{~h}$ the synthesis of 6-methyl-NQ is complete. Therefore, in further studies, the one-pot process was conducted for $30 \mathrm{~h}$ at room temperature. 


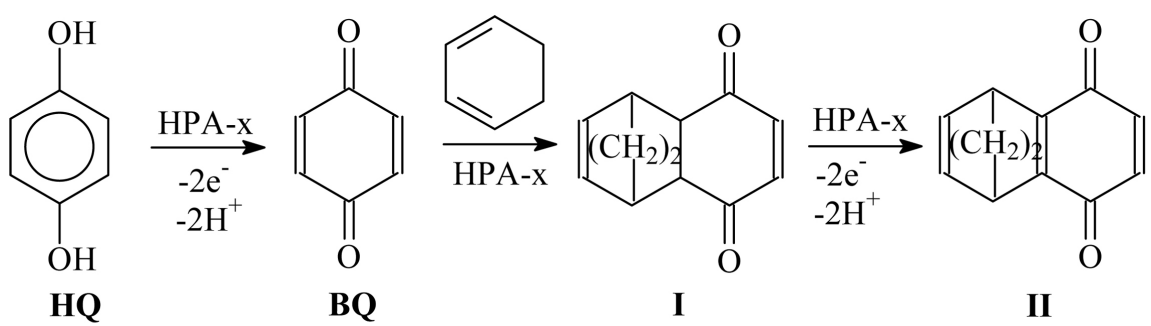

Scheme 2. Reaction between HQ and 1,3-cyclohexadiene.

Table 1. Dependence of parameters of 6-methyl-NQ synthesis on the composition of HPA-x.

\begin{tabular}{ccccc}
\hline HPA-x & $\begin{array}{c}\text { Eof solutions } \\
\text { HPA-x, } \mathrm{V}\end{array}$ & $\begin{array}{c}\text { Molar ratio } \\
\mathrm{V}: \mathrm{HQ}\end{array}$ & $\begin{array}{c}\text { NQ content } \\
\left(\mathrm{C}_{\mathrm{NQ}}\right), \%\end{array}$ & $\begin{array}{c}\text { NQ yield } \\
\left(Y_{\mathrm{NQ}}\right), \%\end{array}$ \\
\hline $\mathrm{H}_{17} \mathrm{P}_{3} \mathrm{Mo}_{16} \mathrm{~V}_{10} \mathrm{O}_{89}(\mathrm{x}=10)$ & 1.16 & $12.5: 1$ & 83 & 67 \\
$\mathrm{H}_{15} \mathrm{P}_{4} \mathrm{Mo}_{18} \mathrm{~V}_{7} \mathrm{O}_{89}(\mathrm{x}=7)$ & 1.09 & $10.5: 1$ & 84 & 65 \\
$\mathrm{H}_{7} \mathrm{PMo}_{8} \mathrm{~V}_{4} \mathrm{O}_{40}(\mathrm{x}=4)$ & 0.995 & $6: 1$ & 98 & 62.5 \\
\hline
\end{tabular}

Conditions: $10 \mathrm{~mL} 0.25 \mathrm{M}$ water solution of HPA-x, volume ratio dioxane:HPA-x = 1:1, reaction time $30 \mathrm{~h}$, $25^{\circ} \mathrm{C}$; molar ratio isoprene: $\mathrm{HQ}=1.25: 1$; complete conversion $\mathrm{HQ}$ and $\mathrm{BQ}$.

Table 2. Dependence of the parameters of 6-methyl-NQ synthesis on the solvent nature.

\begin{tabular}{ccc}
\hline Solvent & NQ content $\left(\mathrm{C}_{\mathrm{NQ}}\right), \%$ & NQ yield $\left(\mathrm{Y}_{\mathrm{NQ}}\right), \%$ \\
\hline 1,4-dioxane & 98 & 62.5 \\
Water & 80 & 54 \\
\hline
\end{tabular}

Conditions: $10 \mathrm{~mL}$ of $0.25 \mathrm{M}$ water solution HPA-4, molar ratio isoprene:HQ = 1.25:1, volume ratio dioxane:HPA- $4=1: 1$, reaction time $30 \mathrm{~h}, 25^{\circ} \mathrm{C}$, complete conversion of $\mathrm{HQ}$ and $\mathrm{BQ}$.

Table 3. Dependence of parameters of 6-methyl-NQ synthesis on time.

\begin{tabular}{ccc}
\hline Time, h & NQ content $\left(\mathrm{C}_{\mathrm{NQ}}\right), \%$ & NQ yield $\left(\mathrm{Y}_{\mathrm{NQ}}\right), \%$ \\
\hline 6 & $5^{\mathrm{a}}$ & 3.5 \\
24 & 84 & 58 \\
30 & 98 & 62.5 \\
\hline
\end{tabular}

Conditions: $10 \mathrm{~mL} 0.25 \mathrm{M}$ water solution HPA-4, volume ratio dioxane:HPA-4 = 1:1, molar ratio isoprene:HQ $=1.25: 1,25^{\circ} \mathrm{C} .{ }^{a}$ According to HPLC and GCMS data main product is 6-methyl-DHNQ $(\mathrm{m} / z$ 174).

At last the catalyst (HPA-4, conditions see Table 1 ) was 8 times reused after regeneration (as described in [8]) without changing yield (61\% - 62.5\%) of 6-methyl-NQ.

The obtained data show that in the presence of HPA-x solution and hydrophilic organic solvent it is possible to synthesize 6-methylnaphthoquinone from HQ and isoprene with a good yield. As we expected, the strongest influence on the process parameters at room temperature is brought by the reaction time as well as by the presence of a hydrophilic organic solvent. Note that in all conducted experiments (except the reaction in the absence of solvent) the reaction product featured neither HQ nor BQ, i.e., the reaction of diene synthesis be- 
tween BQ and diene was completed.

It was important to estimate the possibility to carry out a similar process for other dienes. For comparison, the processes were conducted with the use of HPA-4 and HPA-7 solutions. The results are given in Table 4 and Table 5.

Good results were obtained for the reaction of HQ with trans-piperylene (trans-1-methylbutadiene). In this case, if HPA-4 is used, the pure 5-methyl-NQ is obtained with a yield of $62 \%$. If HPA-7 is used, 5-methyl-NQ is obtained with a yield of $81 \%$, but the content of impurities is increased by $6 \%$.

In the reaction of HQ with 2.3-dimethylbutadiene (DMB), the product contains, besides the corresponding NQ, an admixture of the derivatives of anthraquinone (from $10 \%$ to $48 \%$ ). While a decrease of the molar ratio DMB:HQ to strictly stoichiometric one allows increasing the NQ content to $92 \%$, but an admixture of the AQ derivatives still remains (Table 5).

In the case of the reaction of HQ with an excess of 1,3-butadiene (even at room temperature) the main product of the reaction is $1,4,4 \mathrm{a}, 9 \mathrm{a}$-tetrahydroanthraquinone (THAQ). It is most likely due to the high excess of butadiene (the process was conducted in its atmosphere) that provides a deeper course of diene synthesis when the forming NQ interacts with 1,3-diene. In this case the content of unsubstituted NQ in the solid product does not exceed $45 \%$. At stoichiometric ratio, NQ and 1,3-butadiene (1:1) pure NQ is obtained with a yield of $51 \%$ (Table 4).

Table 4. Reaction of HQ with various dienes in the presence of 0.25 M HPA-4.

\begin{tabular}{cccc}
\hline Diene & $\begin{array}{c}\text { Molar ratio } \\
\text { diene:HQ }\end{array}$ & $\begin{array}{c}\text { NQ content } \\
\left(\mathrm{C}_{\mathrm{NQ}}\right), \%\end{array}$ & NQ yield $\left(\mathrm{Y}_{\mathrm{NQ}}\right), \%$ \\
\hline DMB & $1.2: 1$ & $52^{\mathrm{a}}$ & 38 \\
Piperylene & $1: 1$ & 97 & 62 \\
1,3 -butadiene, atmosphere & $>1: 1$ & $12^{\mathrm{b}}$ & 6 \\
1,3-butadiene, & $1: 1$ & 99 & 51 \\
1,3 -cyclohexadiene & $1: 1$ & $87^{\mathrm{c}}$ & $84^{\mathrm{c}}$ \\
\hline
\end{tabular}

Conditions: $10 \mathrm{~mL} 0.25 \mathrm{M}$ water solution HPA-4, volume ratio HPA-4:dioxane $=2: 1$, reaction time $30 \mathrm{~h}$, RT, complete conversion HQ and BQ. ancluding the admixture of two derivatives AQ. ${ }^{\mathrm{b}}$ According to HPLC and GCMS data main product is THAQ $(70 \%, \mathrm{~m} / \mathrm{z} 212)$, the rest is NQ and DHAQ. 'Adduct of diene synthesis I.

Table 5. Reaction of HQ with various dienes in the presence of 0.25 M HPA-7.

\begin{tabular}{cccc}
\hline Diene & $\begin{array}{c}\text { Molar ratio } \\
\text { diene:HQ }\end{array}$ & $\begin{array}{c}\text { NQ content } \\
\left(\mathrm{C}_{\mathrm{NQ}}\right), \%\end{array}$ & NQ yield $\left(Y_{\mathrm{NQ}}\right), \%$ \\
\hline DMB & $1.2: 1$ & 76 & 71.5 \\
DMB & $1: 1$ & 92 & 77 \\
Piperylene & $1: 1$ & 94 & 81 \\
1,3-butadiene, atmosphere & $>1: 1$ & $45^{\mathrm{a}}$ & 41 \\
1,3 -cyclohexadiene & $1: 1$ & $89^{\mathrm{b}}$ & $88^{\mathrm{b}}$ \\
\hline
\end{tabular}

Conditions: $10 \mathrm{~mL} 0.25 \mathrm{M}$ water solution HPA-7, volume ratio HPA-7:dioxane $=1: 1$, reaction time $30 \mathrm{~h}$, $25^{\circ} \mathrm{C}$, complete conversion of HQ and BQ. ${ }^{\text {PPlus }} 40 \%$ THAQ. ${ }^{\mathrm{b}}$ Adduct of diene synthesis I. 
It was interesting to estimate the possibility to realize the one-pot process with the interaction of HQ with 1,3-cyclohexadiene. It was found out that in this case, the main products of the reaction are monoadduct of the diene synthesis $\mathrm{I}$ ( $M=$ 188 ) and unidentified product with $M=238$. We should note that $M$ of product II of oxidation of adduct of the diene synthesis is 186 , whereas the adduct with 2 moles of diene $(M=268)$ forms only in trace amounts (less than $1 \%)$. The increase in the reaction temperature above RT leads not to the increase in the content of adduct with two moles of 1,3-cyclohexadiene but the polymerization of the latter and resinification of the reaction mixture. The product II did not form in the course of the process at neither room nor elevated temperature, most likely because in this case, no ring aromatization occurs with the oxidation of adduct of the Diels-Alder reaction $\mathrm{I}$.

\section{Conclusions}

In this work we have studied the one-pot synthesis of a number of 1.4-naphthoquinones by interaction of HQ with various 1,3-diene in the presence of water solutions Mo-V-P heteropolyacids HPA-x $(\mathrm{x}=4,7,10)$ as bifunctional catalysts (acid catalysts of the diene synthesis and catalysts of oxidation of HQ and forming adducts) at room temperature. It is shown that under such mild conditions the strongest influence over the parameters of the process is brought by its duration as well as by the presence of a hydrophilic organic solvent, such as 1,4-dioxane.

We have demonstrated the possibility to use this one-pot method to synthesize pure unsubstituted NQ, 6- and 5-methylnaphthoquinones with the yields of $50 \%-80 \%$, as well as 6,7 -dimethylnaphthoquinone of $92 \%$ purity with the yield of $77 \%$.

Before our works, the methods of one-pot synthesis of NQ from HQ have not been described. The results obtained allow to significantly simplify the synthesis of substituted NQ directly from HQ in comparison with the described similar methods.

The possibility of a fast regeneration of the solutions of HPA-x with oxygen in a separate stage opens a prospect of the industrial realization of the processes of this type.

\section{Acknowledgements}

This work was supported by Ministry of Science and Education of Russian Federation (project No. AAAA-A17-117041710081-1).

\section{Conflicts of Interest}

The authors declare no conflicts of interest regarding the publication of this paper.

\section{References}

[1] Grolig, J. and Wagner, R. (2007) Naphthoquinones in Ullmann's Encyclopedia of 
Industrial Chemistry. Wiley-VCH Verlag GmbH \& Co. KGaA, Weinheim.

[2] Daironas, J.V. and Zilfikarov, I.N. (2011) Natural Napthoquinones: Prospects of Medical Application. Marhotin, Shchelkovo.

[3] Huang, G., Zhao, H.-R., Zhou, W., Dong, J.-Y., Zhang, Q.-J., Meng, Q.-Q., Zhu, B.-Q. and Li, S.-S. (2017) 6-Substituted 1,4-Naphthoquinone Oxime Derivatives (I): Synthesisand and Evaluation of Their Cytotoxic Activity. Monatshefte für Chemie-Chemical Monthly, 148, 1011-1023. https://doi.org/10.1007/s00706-016-1899-z

[4] Ju, H.S., Ju, Y.J., Kim, J.E. and Won, J.I. (2001) Preparation of Napthoquinone and Anthraquinone Using Oxidizing Agent. South Korea Patent No. 2001004856.

[5] Ju, H.S., Ju, Y.J., Kim, J.E. and, Won, J.I. (2000) Method of Manufacturing Anthraquinone from 1,4-Napthoquinone and 1,3-Butadiene. South Korea Patent No. 2000001821.

[6] Torres, E., Panetta, C.A., Heimer N.E., Clark B.J. and Hussey C.L. (1991) Synthesis and Properties of 6-(Hydroxymethyl)-9,9,10,10-Tetracyanonaphthoquinodimethane. The Journal of Organic Chemistry, 56, 3737-3739. https://doi.org/10.1021/jo00011a061

[7] Fieser, L.F. and Fieser, M. (1964) Advanced Organic Chemistry. Reinhold Publishing Corporation, New York.

[8] Gogin, L.L., Zhizhina, E.G., Pai, Z.P. and Parmon, V.N. (2015) Prospects of Using Mo-Vphosphoric Heteropolyacid Solutions as Bifunctional Catalysts for Syntheses of Anthraquinones and Their Substituted Derivatives. Russian Chemical Bulletin, 64, 2069-2075. https://doi.org/10.1007/s11172-015-1119-8

[9] Adams, R., et al., Eds. (1949) Organic Reactions. Volume 5, John Wiley \& Sons, New York, 96.

[10] Blatt, A., Ed. (1922) Organic Syntheses. Volume 2, John Wiley \& Sons, New York, 85-86. https://doi.org/10.15227/orgsyn.002.0085

[11] Zhizhina, E.G., Simonova, M.V., Russkih, V.V. and Matveev, K.I. (2005) Catalytic Synthesis of 9,10-Anthraquinone in Presence of Solutions of Mo-V-Phosphoric Heteropolyacid. Kataliz v promyshlennosti, 1, 12-17.

[12] Odyakov, V.F., Zhizhina, E.G. and Maksimovskaya, R.I. (2008) Synthesis of Molybdovanadophosphoric Heteropoly Acid Solutions Having Modified Composition. Applied Catalysis A: General, 342, 126-130. https://doi.org/10.1016/j.apcata.2008.03.008

[13] Heilbron, I.M. and Bunbury, H.M., Eds. (1946) Dictionary of Organic Compounds. Eyre \& Spottiswoode, London. 